\title{
Développement de l'agropastoralisme chez une fraction nomade fixée sur les bords du fleuve Niger au Mali
}

\author{
L. Baudoux ${ }^{1}$ H. Kamil ${ }^{2}$ C.H. Moulin ${ }^{3 *}$
}

\section{Mots-clés}

Nomadisme - Système agropastoral Système de culture - Elevage - Fleuve Niger - Mali.

\begin{abstract}
Résumé
Dans la région de Tombouctou au Mali, les sécheresses des années 1970-80 et la rébellion touarègue des années 1990 ont entraîné la sédentarisation de populations nomades et le développement de l'agriculture. Afin de mieux connaître ces transformations, l'ONG Vétérinaires sans frontières a lancé une série d'études au démarrage d'un nouveau projet d'appui aux organisations agropastorales. L'évolution du système agraire d'une fraction arabe, fixée sur les bords du fleuve Niger, a ainsi été analysée. Trois systèmes de production ont été caractérisés. Les agropasteurs transhumants (20 p. 100 des familles de la fraction) sont sédentarisés sur la frange fluviale. Ils cultivent du riz et du bourgou dans les plaines inondables et possèdent des petits cheptels de ruminants, insuffisants pour satisfaire l'ensemble des besoins de la famille. Les pasteurs nomades (74 p. 100) sont toujours spécialisés en élevage et ont conservé un mode de conduite pastoral reposant sur la mobilité des troupeaux et des familles pour mettre en valeur des parcours sahéliens. Enfin, des agropasteurs semi-nomades (6 p. 100) combinent la vie de sédentaires en saison sèche, sur le site de fixation au bord du fleuve, et le nomadisme en saison des pluies, les travaux des champs étant alors assurés par des salariés. L'étude des relations agriculture élevage et de l'usage des ressources permet de discuter les politiques de développement centrées sur l'intensification agricole sur la frange fluviale.
\end{abstract}

\section{INTRODUCTION}

Les sociétés pastorales sont marquées par deux faits majeurs, la mobilité et la prépondérance de l'élevage dans l'économie familiale $(3,13,16)$. Cependant, les activités agricoles sont rarement absentes et les relations à l'agriculture peuvent prendre des formes très diverses au cours de l'histoire des familles (8). Dans la région de Tombouctou au Mali, d'importants mouvements de populations ont été observés suite aux sécheresses des années 1970-80 et à la rébellion touarègue (1991-94). Ces mouvements et la réorganisation concomitante des systèmes de production avec le développement de l'agropastoralisme ne sont que partiellement connus au travers des interventions d'urgence qui ont eu lieu durant les années 1980 et 1990,

\footnotetext{
1. Cnearc, 1101 avenue Agropolis, BP 5098, 34033 Montpellier Cedex 1, France

2. VSF, 14 avenue Berthelot, 69361 Lyon Cedex 07, France

3. Agro.M, UMR Elevage des ruminants en régions chaudes,

2 place Viala, 34060 Montpellier Cedex 1, France

* Auteur pour la correspondance

Tél. : +33 (0)4 99612365 ; fax : +33 (0)4 67545694

E-mail : moulinch@ensam.inra.fr
}

comme celles menées par l'ONG Vétérinaires sans frontières (VSF). En phase de démarrage d'un nouveau projet, dans un contexte plus serein, VSF souhaitait mieux connaître la situation actuelle des populations nomades par rapport à la sédentarisation et à leurs activités d'agriculture et d'élevage. Le travail présenté ici est le produit d'une des études réalisées en 2003. Il concerne une communauté de pasteurs nomades, sédentarisée sur les rives du fleuve Niger.

Après avoir présenté la zone d'étude et les méthodes de diagnostic utilisées, les trois systèmes de production observés sont caractérisés. Une analyse des relations agriculture élevage à l'échelle de la frange fluviale peut alors être menée. Les limites et intérêts de la démarche d'étude sont discutés avant de dresser les perspectives d'évolution des systèmes de production.

\section{MATERIEL ET METHODES}

La communauté étudiée est une fraction arabe (familles du même segment de lignage patrilinéaire), appelée gouanine. Depuis une cinquantaine d'années, une partie de cette fraction s'est fixée au bord du fleuve Niger à Téherdjé, à 40 km en aval de Tombouctou 
(figure 1). La zone d'étude comprend donc une portion de la frange fluviale autour de Téherdjé, sur environ cinq kilomètres le long du fleuve, que les Gouanines (50 familles) exploitent avec leur voisins kel-tamacheqs, fraction des Kel-Tamouleits blancs (20 familles), et sonraï (190 familles). Cette zone de la frange fluviale est également utilisée à certaines périodes de l'année par une centaine de familles gouanines pratiquant un élevage nomade. Dans ce système nomade, familles et troupeaux se déplacent dans un territoire pastoral correspondant à une bande transversale par rapport au fleuve, allant jusqu'à $80 \mathrm{~km}$ au sud, dans le Gourma (rive droite du fleuve) et $40 \mathrm{~km}$ au nord (rive gauche), ce qui représente une superficie de $600 \mathrm{~km}^{2}$.

Le climat est sahélo-saharien avec des précipitations inférieures en moyenne à $200 \mathrm{~mm} / \mathrm{an}$, survenant de façon irrégulière entre juillet et octobre. Le milieu est constitué de deux zones agroécologiques. L'une correspond aux plaines inondées chaque année par la crue du fleuve (d'août à février), l'autre aux plaines exondées, séparées du lit majeur du fleuve par un cordon de dunes. Dans les plaines inondables, les parties les plus basses, avec des sols vertiques, sont réservées à la culture du bourgou. Les zones plus hautes, avec des sols à structure plus sableuse, sont couramment mises en valeur par une culture de riz flottant. Sur les dunes, des Kel-Tamacheqs et des femmes sonraï pratiquent le maraîchage. Les plaines exondées portent des végétations spontanées utilisées pour le pâturage. Des aménagements hydrauliques (périmètres irrigués villageois) ont été réalisés dans ces plaines exondées, notamment pour la culture du riz, avec utilisation de variétés sélectionnées de riz dressé.

L'étude a été conduite de mai à août 2003 (5), en s'appuyant sur une démarche de diagnostic de systèmes agraires (24) et de systèmes d'élevage (23). La caractérisation des paysages agraires a permis de définir les différentes zones agroécologiques et les systèmes de production qui leur sont liés. Une étude de l'historique des systèmes agraires a été réalisée grâce à des entretiens auprès des chefs de villages ou de sites ainsi que d'anciens et de lettrés. Cette étude historique a permis d'analyser les évolutions des modalités d'accès au foncier et les relations entre les différentes communautés et l'espace. Par la suite, des entretiens ont été menés avec des agropasteurs pour étudier les pratiques d'élevage et de culture. Enfin, 55 enquêtes à questions fermées ont permis de recueillir des données sur les unités de production (actifs familiaux, terres, cheptel) et les unités de consommation (famille), pour les familles résidant dans la frange fluviale. Deux personnes ont réalisé les enquêtes, l'une menant l'entretien et l'autre assurant la prise de notes, assistées d'un auxiliaire vétérinaire assurant la traduction grâce à sa maitrise des différentes langues parlées dans la zone.

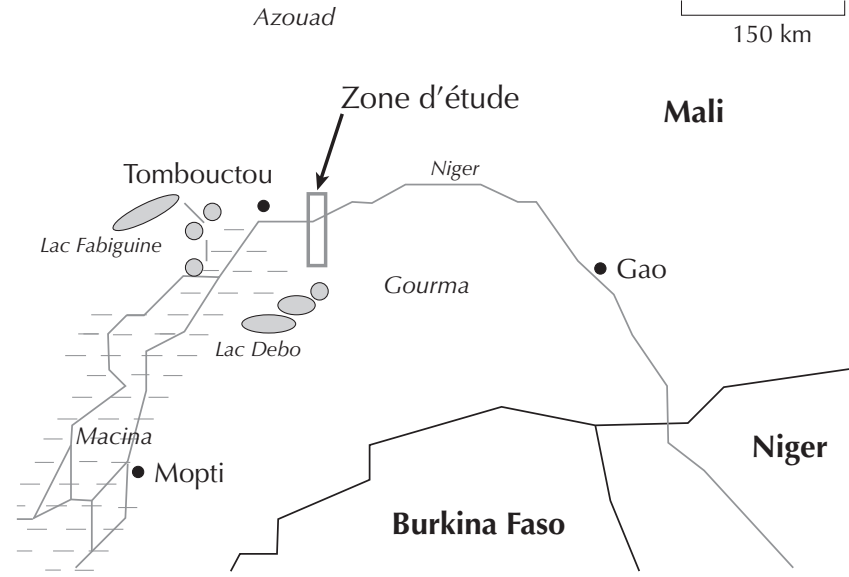

Figure 1 : situation de la zone d'étude dans la boucle du Niger.
L'analyse historique a eu pour but de rassembler un ensemble de faits, d'éléments localisés dans le temps et dans l'espace, qui ont ensuite été ordonnés, au travers des concepts de système agraire et de système de production (12). Une typologie des systèmes de production a ainsi été dressée, sur la base des activités exercées et de la mobilité des troupeaux et des familles. Les données qualitatives et quantitatives issues des entretiens et enquêtes ont alors été utilisées pour caractériser la structure et le fonctionnement de chaque système (6). Pour les activités agricoles, la productivité de la terre et du travail a été évaluée en calculant la valeur ajoutée brute par hectare ou par journée de travail d'un actif. Les familles détenant plusieurs espèces animales, la taille du troupeau a été exprimée en unités de bétail tropical (UBT), en comptant 0,73 UBT pour un bovin et 0,12 UBT pour un petit ruminant (9). Une restitution auprès de représentants de la population et des acteurs locaux du développement de cette région a permis de valider les informations recueillies et les premières analyses.

\section{RESULTATS}

\section{Historique des systèmes agraires dans la frange fluviale}

La mémoire des anciens permet de remonter jusqu'à la moitié du $\mathrm{XIX}^{\mathrm{e}}$ siècle, période pendant laquelle les Kel-Tamacheqs dominaient la région. Les Gouanines étaient alors peu nombreux. En bons termes avec les Kel-Tamouleïts, ils obtinrent un droit d'usage sur un territoire s'étendant de part et d'autre du fleuve. Ils baptisèrent la zone proche du fleuve Téherdjé, siginifiant cadeau en langue tamacheq. Une partie des Haratines, leurs captifs, y furent regroupés pour qu'ils y cultivent et pratiquent la cueillette. En contrepartie, ces derniers devaient leur reverser une rente agricole. Les autres Haratines ont poursuivi les activités qu'ils menaient jusqu'alors : gardiennage des animaux et exhaure de l'eau.

L'équilibre sur lequel reposait ce système a été profondément affecté par la colonisation française dont les premiers effets ont été ressentis à la fin du $\mathrm{XIX}^{\mathrm{e}}$ siècle. Durant la période coloniale et les débuts de l'indépendance (1890-1965), les changements majeurs résidaient dans les modifications des relations entre les communautés et l'arrivée de migrants. Quelques familles sonraï sont arrivées de Gao par le fleuve et ont demandé aux Kel-Tamouleïts et aux Gouanines l'autorisation de s'installer et d'utiliser quelques terres au bord du fleuve. Les ayants droit acceptèrent et des villages se sont peu à peu constitués, avec des populations vivant d'agriculture et des produits de la pêche. Les Haratines qui étaient soumis aux ordres de leurs maîtres ont acquis peu à peu une certaine liberté. Considérée alors comme gouanine, une grande partie, dépourvue de moyens, a poursuivi l'agriculture sur les terres qu'ils cultivaient préalablement. En effet, désormais devenus membres de la fraction, ils ont pu obtenir des droits d'usage sur le territoire des Gouanines. Quelques familles haratines qui avaient les fonds nécessaires ont pu se lancer dans l'élevage pastoral alors soutenu par le gouvernement français (politique d'appui à la santé animale et à la mise en place de puits pastoraux). Privés des produits agricoles, auparavant issus de leurs captifs haratines, les nomades gouanines ont alors développé des échanges monétaires avec les agriculteurs de la zone. A l'indépendance, le gouvernement malien a encouragé la sédentarisation des populations nomades pour en avoir un meilleur contrôle (recouvrement de l'impôt, contrôle des frontières...). Des magasins d'approvisionnement ont ainsi été mis en place pour que les nomades aient un accès plus facile aux denrées agricoles. Selon un chef de fraction, « cette sécurité alimentaire les a encouragés à se sédentariser ». Ainsi, certains Gouanines se sont installés à Téherdjé. 
Les décennies qui suivirent l'indépendance ont été marquées par l'accroissement de l'aridité avec notamment deux crises climatiques, les sécheresses de 1973 et 1984. Par ailleurs, les changements politiques, d'abord favorables aux pasteurs, puis aux populations sédentaires, couplés à l'affirmation de la non-propriété des terres pastorales ont animé un sentiment de révolte chez les nomades qui éclata lors de la rébellion touarègue (1991-94). Enfin, les interventions de multiples acteurs de l'aide au développement ont également contribué au bouleversement de l'équilibre du système agraire.

Face à l'accentuation des risques climatiques, de nombreuses familles gouanines se sont sédentarisées à cette période pour diversifier leurs activités. De plus, cela leur permettait d'obtenir des aides extérieures dans le cadre de plans d'urgences, appuis qu'elles n'auraient pas forcément reçus si elles étaient restées mobiles. Par exemple, les habitants de Téherdjé ont obtenu un financement par le Haut comité aux réfugiés en 1996 pour l'installation d'un périmètre irrigué villageois qui leur a permis de cultiver du blé. Par ailleurs, une partie de la fraction a été déplacée dans des camps de sinistrés ou de réfugiés dans lesquels les Gouanines ont pu apprendre différentes techniques agricoles telle que le maraîchage qu'ils ont mis en pratique dès leur retour.

Plusieurs éléments ont contribué au renforcement du secteur agricole. Tout d'abord, le prix des denrées agricoles a augmenté contrairement au prix de la viande (résultat des sécheresses et des conditions du marché local caractérisé par une faible demande). Cette dégradation des termes de l'échange a encouragé les éleveurs à produire eux-mêmes leur riz. Les interventions de l'aide extérieure ont majoritairement appuyé les projets agricoles, pour assurer la sécurité alimentaire des populations. Cela s'est rapidement traduit par une pression sur l'espace au niveau de la frange fluviale, avec la mise en culture, dans les plaines inondables, de terres jusque-là réservées au pâturage. Ce phénomène s'est accru jusqu'à l'amorce de conflits fonciers.

Parallèlement, les agriculteurs sonraï ont diversifié leurs sources de revenus, grâce à l'aide extérieure. Une partie de l'accroissement de leurs revenus a été accumulée sous forme d'animaux d'élevage, conduits selon un mode d'élevage sédentaire. Aussi, suite à l'orientation commune des populations vers l'agropastoralisme, les échanges commerciaux ont globalement diminué et des relations de concurrence se sont développées entre les systèmes de production des différentes communautés, et cela au sein d'un espace limité.

Les Gouanines ont ainsi développé différents modes de valorisation du milieu, notamment grâce à la situation stratégique de leur territoire d'attache situé sur la frange fluviale, ce qui leur a permis de pratiquer l'agriculture. Trois grands systèmes de production peuvent être distingués aujourd'hui : l'élevage nomade, l'agriculture combinée à un élevage transhumant, système dans lequel les familles sont sédentarisées à Téherdjé (agropasteurs transhumants), et enfin l'agriculture et l'élevage semi-nomade, les familles étant fixées à Téherdjé mais repartant en nomadisme avec leur troupeau pendant la saison humide (agropasteurs semi-nomades).

\section{Systèmes de production des familles de la fraction gouanine}

\section{Elevage nomade}

Même si l'agriculture apparaît être une stratégie intéressante dans un contexte de crise, elle n'a pas été adoptée par toute la fraction gouanine. Les trois quarts des familles ont conservé leurs modes de vie et de production pastoraux en adaptant sans cesse leurs pratiques aux évolutions du milieu. Ces familles détiennent des troupeaux multispécifiques, composés de bovins et de petits ruminants (pour l'alimentation en lait et la vente d'animaux), et d'animaux de transport (ânes et dromadaires). A l'approche de la saison des pluies, ils descendent vers le sud pour profiter des zones d'herbage déjà arrosées par les premières pluies. Puis ils remontent vers le nord et se déplacent de point d'eau en point d'eau pour profiter de la végétation la plus riche et la plus abondante. Pendant la saison sèche, pour une période qui peut durer six mois, les familles se stabilisent autour des puits au sein de leur terroir d'attache, aire géographique dans laquelle la fraction vit la majeure partie de l'année (17), comportant des ressources stratégiques, comme des puits profonds (30). Ceux qui en ont les moyens apportent des compléments alimentaires aux animaux les plus fragiles et aux femelles laitières. La mobilité vers le sud en saison des pluies permet d'assurer le renouvellement des ressources végétales sur les parcours de saison sèche (pas de prélèvement pendant la période de croissance de la végétation).

L'activité d'élevage de ces familles nomades ne suffit pas toujours à assurer leur survie. Ceci dépend du rapport de la taille du troupeau à la taille de la famille. Lorsque la main d'œuvre familiale est excédentaire par rapport aux travaux à réaliser sur le troupeau, des membres de la famille émigrent pour procurer un revenu complémentaire. Les revenus de ces activités extérieures permettent de faire vivre la famille (viabilité à court terme) et de limiter l'exploitation du troupeau pour reconstituer le cheptel suite à un épisode de sécheresse ou des problèmes sanitaires (ce qui assure la viabilité à moyen terme). Les liens de solidarité, très développés au sein de la fraction, permettent la survie des familles ne possédant pas un cheptel de taille suffisante et ne disposant pas de main d'œuvre pour partir travailler à l'extérieur. Une dizaine de ces familles, dans une situation très précaire, a entrepris de faire des cultures de décrue (mil, sorgho) dans une mare temporaire dans le Gourma. Ces familles ont refusé de se rendre à Téherdjé, dont les caractéristiques du milieu sont beaucoup plus propices à la culture, car elles préfèrent conserver leur terroir d'attache et leur mode de vie nomade.

Ces familles pratiquant l'élevage nomade font face à d'importantes difficultés sociales et sanitaires liées au manque d'infrastructures (école, magasin d'approvisionnement, centre de santé, voies de communication...). Les conflits fonciers entre les différentes communautés deviennent de plus en plus fréquents. Les limites des terres ne sont pas matérialisées et le déplacement fréquent des familles les empêche de contrôler l'utilisation que les autres font de leurs aires de parcours, ce qui occasionne des litiges fonciers (principalement avec les Sonrai) dans les zones où l'eau est présente au moins une partie de l'année (frange fluviale, mares temporaires).

\section{Association de l'agriculture et de l'élevage transhumant}

Dans ce système de production, les familles sont constituées de 10 personnes, avec 2,25 actifs en moyenne. Elles résident toute l'année à Téherdjé où elles pratiquent l'agriculture. Les surfaces moyennes cultivées par actif sont de 0,31 ha pour le riz flottant et 0,19 ha pour le bourgou. Ces familles détiennent également des animaux, avec en moyenne 5,4 UBT par famille. Le cheptel moyen, en proportion des UBT totales, est composé de 25 p. 100 de bovins, 15 p. 100 d'ovins et 60 p. 100 de caprins, et 60 p. 100 des familles possèdent des ânes. Ces animaux sont conduits selon un mode d'élevage agropastoral reposant sur l'utilisation de ressources spontanées et cultivées (figure 2). Pendant la saison des pluies, les troupeaux sont confiés à d'autres familles, nomades ou semi-nomades, pour aller vers le sud profiter des pâturages arrosés par les premières pluies. Ce mouvement du bétail, alors que la famille reste pour cultiver à Téherdjé, s'apparente à une transhumance. Il permet d'éloigner les animaux des champs pendant la période de culture et de leur faire profiter des pâturages riches et abondants du Gourma en saison des pluies. Pendant cette période, quelques animaux sont gardés avec la famille à Téherdjé : ils assurent la production laitière et 


\begin{tabular}{|c|c|c|c|c|c|c|c|c|c|c|c|c|c|}
\hline & Mars & Avr. & Mai & Juin & Juil. & Août & Sept. & Oct. & Nov. & Déc. & Janv. & Fév. & Mars \\
\hline Saisons & \multicolumn{4}{|c|}{ Saison sèche chaude } & \multicolumn{4}{|c|}{ Saison des pluies } & \multicolumn{4}{|c|}{ Saison sèche froide } & \\
\hline Crue & & & & & & & & & & & & & \\
\hline
\end{tabular}

Frange fluviale

Bourgoutières

\begin{tabular}{|l|l|l|l|l|l|l|l|l|l|l|l|}
\hline Rizières & & & & & & & & & \\
\hline
\end{tabular}

\section{Gourma}

Pâturage naturel

\section{Aliments distribués}

Bourgou séché, aliments du bétail

Figure 2 : calendrier alimentaire et fourrager des troupeaux chez les agropasteurs transhumants.

répondent à d'éventuels besoins monétaires. Le reste de l'année (d'octobre à juin), l'ensemble du troupeau divague autour de Téherdjé, sur différents espaces selon l'avancée de la décrue. En raison de l'insuffisance des pâturages aux alentours des habitations et de la charge animale élevée, les agropasteurs apportent des compléments alimentaires pour le cheptel en saison chaude.

La taille de troupeau minimum pour qu'une famille puisse vivre de la seule activité d'élevage a été déterminée d'après les déclarations des familles gouanines fixées à Téherdjé. Pour une famille de 10 personnes, il faudrait au moins 10 bovins, 60 petits ruminants et 2 ânes, soient 15 UBT. Le seuil de survie pour une telle famille, c'est-à-dire le revenu nécessaire pour assurer les besoins minimaux pour l'alimentation, l'habillement et la santé, a été estimé à $1120000 \mathrm{Fcfa}$ (environ $1700 €$ ). Un calcul théorique du revenu permis par le troupeau de 15 UBT (avec autoconsommation de lait et vente d'animaux dans la limite du renouvellement du stock de reproductrices) donne un niveau de revenu de l'ordre du seuil de survie. Ces calculs permettent de recouper les déclarations sur la taille minimale du troupeau.

Ces familles d'agropasteurs transhumants détiennent des troupeaux de taille largement inférieure au troupeau minimum (5,4 UBT contre 15). Elles perçoivent l'agriculture comme indispensable à leur survie. Le vice-président des éleveurs de Téherdjé en témoigne ainsi : «On préfère nos animaux et même leurs déjections à l'agriculture, mais honnêtement, c'est la seule solution aujourd'hui pour s'en sortir. J'aime mes animaux, je les accompagnerai jusqu'à leur dernière demeure. Alors, je changerai mon bâton avec une houe ». Cependant, le troupeau assure de multiples fonctions dans ce système agropastoral : production de viande et de lait, épargne et assurance.

Ce système de production est le plus représenté à Téherdjé $(80$ p. 100 des familles) mais il ne concerne que 20 p. 100 de l'ensemble des familles de la fraction. Ces familles sédentaires développent de nouvelles relations avec l'extérieur. Elles peuvent bénéficier de services sociaux tels que l'éducation ou la santé. Des familles nomades confient d'ailleurs leurs enfants à leurs cousins fixés à Téherdjé afin d'assurer leur scolarisation. Ces agropasteurs se lancent également dans d'autres activités, comme le commerce, et si la main d'œuvre est excédentaire, certains membres de la famille partent en émigration.

\section{Association de l'agriculture et de l'élevage semi-nomade}

Dans ce système de production, les familles conjuguent la vie de sédentaire à Téherdjé pendant la saison sèche et la vie de nomade en saison des pluies. En juin, les familles partent vers le sud avec leurs troupeaux, puis elles remontent alors peu à peu vers le fleuve qu'elles atteignent aux environs du mois de septembre. Les animaux sont alors laissés sur des zones de pâturage proches du village tandis que les familles reprennent leur vie de sédentaires. Elles peuvent assurer la récolte du riz et du bourgou en complétant généralement leur main d'œuvre par des ouvriers agricoles (généralement des Bellahs, descendants des anciens captifs des KelTamacheqs). Les autres opérations culturales, à réaliser lorsque les familles sont parties dans le Gourma (labour, semis, désherbage ou encore repiquage du bourgou), sont déléguées à un membre d'une famille sédentaire qui embauche des salariés temporaires pour réaliser les travaux.

Peu de familles gouanines (6 p. 100) mettent en œuvre ce système de production. Elles exploitent le milieu en tirant partie à la fois de l'agriculture et de l'élevage. Ces familles disposent a priori d'un cheptel suffisant pour se spécialiser dans l'élevage, avec 20 à 50 UBT par famille. Le troupeau est composé de 60 p. 100 de bovins, 25 p. 100 d'ovins et 15 p. 100 de caprins. Cependant, elles pratiquent l'agriculture, notamment pour limiter les prélèvements sur les troupeaux. Les surfaces cultivées sont assez faibles, avec 0,2 ha cultivé par actif alors qu'un actif chez les agropasteurs transhumant cultive 0,5 ha. La part des surfaces consacrée au bourgou (50 p. 100) montre l'intérêt accordé à la production de fourrages pour les troupeaux. La culture a également une autre utilité plus discrète : celle de marquer les terres afin d'éviter que les voisins sonraï ne se les approprient (selon les dires des habitants de Téherdjé). Il faut signaler que ces familles, dont les systèmes de production dégagent les revenus les plus élevés, disposent en plus des revenus tirés du travail de membres de la famille émigrés.

\section{Relations agriculture élevage}

\section{Intégration agriculture élevage dans les unités de production}

La traction animale et la fumure organique sont deux thèmes techniques majeurs de l'intégration agriculture élevage dans les unités de production (22). Si les ânes et les dromadaires sont utilisés pour 
le transport (notamment au moment des récoltes), la culture attelée, en revanche, est pratiquement absente chez les agropasteurs de Téherdjé. En effet, un grand nombre de travaux agricoles sont réalisés manuellement par des salariés temporaires. Ces salariés agricoles sont des Bellahs, résidant dans un village voisin. N'ayant pas accès à la terre, ils vendent leur force de travail pour se nourrir. Dans un tel contexte d'offre de main d'œuvre et de concurrence sur les espaces pour la mise en culture, l'intérêt de la culture attelée n'est pas évident. L'utilisation des déjections animales comme fumure organique est également très faible. Les sols sableux des dunes étaient fertilisés avec des déjections animales lorsque les agropasteurs pratiquaient le maraîchage. Mais ces cultures ont été abandonnées du fait des dégradations causées par le bétail. Dans les plaines inondables, le maintien de la fertilité est assuré en partie par les alluvions apportées par la crue du fleuve : l'intérêt des apports de déjections animales n'est pas net par rapport au travail nécessaire pour rassembler et transporter les déjections. Enfin, les Gouanines ne cultivent pas forcément le même champ d'une année sur l'autre. Cette réglementation foncière décourage l'investissement des producteurs quant à l'entretien de la fertilité de leur sol.

\section{Développement de la riziculture chez les agropasteurs de Téherdjé}

Pour conforter la base agricole des systèmes agropastoraux, l'aménagement de périmètres irrigués villageois dans les plaines exondées permettrait une extension des surfaces cultivées. Ce thème de développement a été largement appuyé par les coopérations extérieures et adopté par les agropasteurs sonraï des villages voisins. La culture du riz dans ces périmètres, avec des variétés améliorées de riz dressé, assurerait une meilleure sécurité alimentaire des familles gouanines et permettrait de conforter l'élevage en consacrant plus de surfaces au bourgou dans les plaines inondables (substitution du bourgou au riz flottant). Mais cette répartition spatiale des cultures pose plusieurs difficultés qui expliquent que les agropasteurs de Téherdjé maintiennent la culture du riz flottant dans les plaines inondables. Tout d'abord, la rémunération d'une journée de travail agricole est supérieure pour le riz flottant en plaines inondables par rapport au riz dressé en périmètre irrigué : 250 à 1300 Fcfa par journée de travail pour le riz dressé, selon le climat de l'année, contre 500 à $1800 \mathrm{Fcfa}$ pour le riz flottant. La gestion des périmètres irrigués suppose également une organisation commune relativement complexe, avec mise en place d'un comité de gestion qui coordonne les opérations culturales réalisées en commun (pépinière, repiquage par exemple) et fait fonctionner l'installation (fonctionnement et entretien des motopompes) en percevant une redevance. Enfin le riz flottant, de par la taille de ses tiges, procure de la paille en quantité importante, valorisée par les activités d'élevage.

\section{Agriculture, élevage et gestion des ressources dans la frange fluviale}

Les bords du Niger sont un lieu privilégié pour l'élevage : le fleuve constitue un point d'eau permanent et irrigue de nombreuses plaines favorables au développement de la végétation et ainsi au pâturage. Par ailleurs, en période de crue, la frange fluviale devient un bassin de production de plantes fourragères grâce au bourgou. Ainsi, dès le mois de septembre et pendant toute la décrue, elle est le point de rencontre des éleveurs nomades. Mais c'est également un espace privilégié pour la production agricole, sous ce climat sahélo-saharien rendant très aléatoire la culture pluviale.

L'accroissement de l'aridité qui sévit depuis les années 1970 est particulièrement ressenti dans la zone d'étude. La crue du fleuve n'est plus aussi importante qu'avant, en raison des barrages et des aménagements en amont. De ce fait, les bras du Niger qui irriguaient les plaines au-delà des dunes ont disparu. Les surfaces cultivables ont ainsi diminué de façon importante. Par ailleurs, la sédentarisation des populations nomades, couplée à l'accroissement de la population sonraï, a entraîné un intérêt croissant pour la production agricole, également encouragée par l'aide extérieure. Ces deux phénomènes ont exacerbé la pression sur les terres cultivables. Parallèlement à l'augmentation des activités agricoles, la charge animale entretenue en bordure du fleuve a augmenté, en raison de la sédentarisation d'une partie de la fraction gouanine mais également de l'accroissement du cheptel des Sonraï (agriculteurs devenant agropasteurs).

La concurrence sur les espaces et les ressources se fait donc de plus en plus vive et génèrent deux types de conflits. Ce sont tout d'abord des conflits fonciers, pour l'accès aux surfaces cultivables ou pour maintenir des surfaces non cultivées afin de disposer de pâturages de décrue. Des conflits opposent ainsi sédentaires et éleveurs nomades ou semi-nomades. D'une manière générale, ces derniers se plaignent de l'extension des cultures que revendiquent les sédentaires comme un besoin vital pour faire face à l'augmentation démographique. L'autre source majeure de conflits relèvent des tensions liées à la pratique conjointe de l'agriculture et de l'élevage sur ces espaces. Ce sont les cultures qui règlent le rythme de déplacements des animaux : les producteurs se sont organisés de manière à ce que les plaines inondables soient libérées de la plus grande partie des animaux pendant le temps des cultures. Ces derniers peuvent en bénéficier le reste de l'année. Ainsi, le riz est semé quand les animaux des sédentaires et semi-nomades sont partis dans le Gourma. Quand ils reviennent, les cultures sont protégées par la crue. Mais ces règles d'usage ne sont plus toujours respectées. Les risques de dégradation des cultures par les animaux sont plus ou moins forts selon les périodes de l'année (tableau I). La gestion des bourgoutières est particulièrement difficile, le repiquage ayant lieu pendant

\section{Tableau I}

Risque de conflits entre agriculture et élevage au cours de l'année

\begin{tabular}{|c|c|c|c|c|}
\hline & Avr.-juin & Juil.-août & Sept.-déc. & Janv.-mars \\
\hline Elevage & $\begin{array}{l}\text { Forte concentration } \\
d^{\prime} \text { animaux dans } \\
\text { la frange fluviale }\end{array}$ & $\begin{array}{l}\text { Déplacements } \\
\text { dans le Gourma }\end{array}$ & $\begin{array}{l}\text { Remontée progressive } \\
\text { vers la frange fluviale }\end{array}$ & $\begin{array}{l}\text { Pâturage des résidus } \\
\text { du riz puis du bourgou }\end{array}$ \\
\hline Agriculture & $\begin{array}{l}\text { Repiquage } \\
\text { du bourgou }\end{array}$ & $\begin{array}{l}\text { Labour, semis du riz, } \\
\text { récolte du mil }\end{array}$ & $\begin{array}{l}\text { Entretiens et } \\
\text { récolte du riz }\end{array}$ & Récolte du bourgou \\
\hline $\begin{array}{l}\text { Risques de dégâts } \\
\text { sur les cultures }\end{array}$ & $\begin{array}{l}\text { Elevés sur } \\
\text { les bourgoutières }\end{array}$ & Modérés & Modérés à élevés & Faibles \\
\hline
\end{tabular}


la période de soudure pour les animaux (juin). Les dégâts aux cultures peuvent être importants et sont avancés comme raison de l'arrêt de la production de blé et du maraîchage à Téherdjé. Mais la présence des animaux pendant une grande partie de l'année pose d'autres problèmes. Les animaux ne sont ni gardés, ni parqués (hormis quelques exceptions). Ils divaguent ainsi autour du village, notamment sur les dunes où des signes d'érosion sont relevés. Le sable n'y est pas maintenu et vient se répandre sur les plaines inondables amoindrissant leur capacité de rétention d'eau. Selon les dires des cultivateurs, la fertilité de ces terres est très sensible à l'ensablement et les rendements agricoles s'en trouvent réduits.

\section{DISCUSSION}

\section{Limites, intérêts et perspectives de la méthode}

Les résultats présentés ici sont le produit d'une étude de courte durée : deux mois et demi de travail effectif d'observations et d'enquêtes sur la zone d'étude, avec peu de moyens humains. Dans ces conditions logistiques, les activités agricoles ont été quantifiées de façon satisfaisante. En revanche, pour l'élevage, il n'a pas été possible de quantifier certains éléments, notamment ceux qui concernent le foncier pastoral et le peuplement animal global. L'augmentation de la charge animale dans la frange fluviale n'a ainsi pas pu être calculée, ce qui n'a pas permis d'apprécier l'ampleur de ce phénomène pourtant rapporté par différentes personnes au cours des entretiens.

Les enquêtes quantitatives sur les systèmes de production n'ayant pas pu être effectuées chez les familles nomades, les tailles des familles et des troupeaux ne sont pas connues pour ce système de production. Il n'a pas ainsi été possible d'évaluer la proportion de familles nomades se situant en dessous du seuil de survie.

Malgré ces limites, la méthode utilisée a permis de repérer les différents systèmes de production agricole, d'expliquer les modes actuels de mise en valeur de ce milieu sahélien et de pointer les enjeux autour des usages de ressources clés, localisées dans la frange fluviale, déterminants pour l'avenir de cette fraction gouanine. Cette démarche systémique a permis d'articuler de façon pertinente les informations qualitatives et quantitatives. Elle a consisté à repérer tout d'abord des types d'unités de production à partir de l'étude historique et des données qualitatives sur les activités et les pratiques mises en œuvre. Les éléments quantitatifs ont été utilisés dans un deuxième temps, lorsqu'ils ont pu être recueillis, pour caractériser les différents systèmes identifiés. Cette démarche a donc été très différente des méthodes d'étude de la diversité des unités de production à partir d'un ensemble de variables traitées au moyen d'analyses multivariées $(2,20,26)$. Elle répondait mieux aux objectifs, notamment parce qu'elle s'appuyait sur une théorie du fonctionnement des systèmes analysés, comme cela a déjà été discuté par Landais (21).

Dans cette situation d'élevage pastoral, les équilibres entre activités (élevage, agriculture, émigration) et entre taille de la famille et taille du troupeau peuvent être rapidement déplacés, sous l'effet de facteurs internes aux familles (modifications de la composition du groupe familial) ou d'événements extérieurs (sécheresse), comme le souligne Thébaud (31) à propos des pasteurs peuls du Niger oriental. Il faut donc prêter une attention toute particulière aux évolutions récentes des activités des familles. Pour la mise en œuvre de diagnostic agraire dans les régions d'élevage pastoral mobile, l'approche historique, centrée sur l'identification des systèmes agraires successifs sur le long terme (un siècle et demi), pourrait donc être utilement complétée par un recueil d'informations sur la trajectoire récente des unités de production, comme cela a été développé dans des études récentes sur les systèmes d'élevages de petits ruminants dans le sud de la France $(25,27)$.

\section{Viabilité des systèmes de production et itinéraires des familles}

La viabilité du système pastoral spécialisé n'a pas pu être évaluée pour les familles nomades. Cependant la précarité d'une dizaine de familles réalisant des cultures de décrue dans des mares temporaires et les phénomènes de migration procurant des revenus extérieurs étaient les signes des difficultés économiques d'une partie au moins de ces familles. Ce résultat est concordant avec d'autres études menées sur l'élevage pastoral en Afrique subsaharienne (1, $14,31)$.

L'agriculture est une voie possible de diversification permettant la sécurisation des systèmes de production. Le cadre d'analyse proposé par Bonfiglioli (8) s'applique assez bien à la situation de la fraction gouanine ; il permet de saisir les fonctions de l'agriculture et de faire des hypothèses sur les trajectoires des familles entre les trois systèmes de production observés.

Bonfiglioli distingue trois configurations d'agropastoralisme pour les familles à l'origine spécialisées en élevage : agropastoralisme d'attente, de sécurité ou d'opportunisme. Les éleveurs nomades pratiquant des cultures pluviales dans le Gourma pourraient être en attente; des conditions plus favorables (augmentation de la taille du troupeau ou augmentation de la main d'œuvre familiale pour envoyer un membre de la famille en émigration) leur permettraient d'arrêter l'agriculture. Dans le cas contraire, ces familles pourraient être amenées à quitter leur mode de vie pastoral. En revanche, les agropasteurs transhumants, fixés à Téherdjé, pratiquent un agropastoralisme de sécurité. Les conditions favorables aux cultures dans la frange fluviale fournissent une base agricole solide pour construire un système agropastoral viable (31). Ces agropasteurs peuvent être d'anciens Haratines qui pratiquent l'agriculture depuis longtemps ou d'anciens nomades qui ont perdu tout leur cheptel lors des crises des années 1970-90. Il semble peu probable que ces familles adoptent à nouveau un système de production nomade.

Bonfiglioli propose une troisième configuration, l'agropastoralisme d'opportunisme, avec une double inscription spatiale pour les pasteurs, entre brousse et le village, sans que l'agriculture soit forcément pratiquée. Ici, les agropasteurs semi-nomades sont dans une configuration agropastorale plus complète, avec marquage des terres par la culture, phénomène observé ailleurs, comme par exemple chez les éleveurs des steppes semi-arides du Maghreb (10). Mais seule une partie des travaux agricoles sont réalisés par la famille, essentiellement la récolte, ce qui les rapproche des pratiques de cueillette classique chez les pasteurs sahéliens (7). Ce système de production peut être interprété comme une forme de transition pour des transhumants qui, ayant réussi à accumuler suffisamment de cheptel, reprennent un nomadisme de saison des pluies, avant un retour vers un nomadisme intégral. C'est peut-être aussi une nouvelle configuration durable d'agropastoralisme, maintenant un équilibre entre un mode de vie pastoral et l'accès à un environnement socio-économique de sédentaires sur la frange fluviale. Ce système de production présente une bonne viabilité économique ; il est cependant pratiqué par peu de familles aujourd'hui. Il repose en effet sur l'accès à des ressources foncières rares, dans les plaines inondables sur la frange fluviale. Il ne peut guère constituer un modèle pour toutes les familles spécialisées en élevage.

\section{Relations entre systèmes de production}

Si les familles peuvent passer d'un système de production à un autre, il faut aussi noter la dépendance des systèmes entre eux. La 
conduite de l'élevage aujourd'hui chez les agropasteurs transhumants dépend de la présence de nomades (ou semi-nomades) qui prennent une partie des animaux des sédentaires pour les emmener dans le Gourma en saison des pluies. Les cheptels étant de petite taille, il n'y aurait en effet pas intérêt à mobiliser un membre de la famille pendant 3-4 mois pour emmener les animaux en transhumance, mais il y pourrait être intéressant de regrouper les cheptels des sédentaires pour constituer un troupeau de transhumance dont la garde serait à la charge de l'ensemble des familles. Les agropasteurs semi-nomades dépendent de la présence des sédentaires : ceux-ci embauchent les salariés temporaires pour faire les travaux des champs des semi-nomades, ils accueillent également les jeunes pour assurer la continuité de leur scolarisation. Certaines familles nomades passent des arrangements avec des sédentaires pour cultiver du bourgou, le fourrage étant transporté vers le Gourma, grâce à des dromadaires, et distribué en complément aux ruminants pendant la saison sèche chaude. Ces liens entre les familles mettant en œuvre différents systèmes de production permettent ainsi une dissociation de la mobilité des troupeaux et des familles $(4,19)$, assurant un accès plus facile, pour une partie de la population, à un ensemble de services.

Pour sécuriser ce système agraire, il convient de promouvoir des actions qui prennent en compte ces complémentarités et raisonnent la mise en valeur à la fois des zones de pâturages exondées et de la frange fluviale. Le maintien de la mobilité des troupeaux est indispensable à une mise en valeur efficace des zones exondées $(11,28)$, la mobilité ayant un rôle important dans les stratégies antirisques $(15,31)$. La sécurisation de l'élevage mobile reste une priorité dans les différentes politiques de développement. L'augmentation de la production fourragère, avec la culture du bourgou dans les zones inondables, pourrait être une voie de sécurisation en améliorant l'alimentation animale. Cependant, les premiers animaux concernés seraient certainement ceux qui restent en bordure du fleuve : l'accès aux bourgoutières ou aux lieux de stockage du bourgou y est aisé. Les sédentaires et semi-nomades profiteraient ainsi de cette intensification. Les bénéfices qu'en tireraient les nomades seraient certainement moindres puisqu'ils n'ont pas accès librement à la frange fluviale. Il faudrait donc travailler sur les règles d'accès à ces ressources fourragères, ce qui renvoie au problème du contrôle du foncier au sein de la fraction ou au développement du marché des fourrages.

\section{Gestion de l'espace par les différentes communautés}

Les communautés exploitant la frange fluviale (gouanine, sonraï, kel-tamacheq) maîtrisent les techniques agricoles et d'élevage. Elles se sont organisées par le passé de manière informelle pour que chacun de leurs membres ait accès aux ressources de manière équitable et durable. Cependant, l'entente entre ces communautés est menacée ; nombre de conflits, notamment fonciers, les opposent déjà les unes aux autres. Les populations tentent de protéger leur territoire et cherchent à récupérer les conventions qui leur attribuaient des droits d'usage au temps de la colonisation. Dans ce contexte, les services d'appui au développement doivent plutôt résider dans le secteur institutionnel $(18,28)$. Les organisations coutumières doivent trouver une personnalité juridique, dans la mesure où elles sont capables de répondre aux exigences de l'administration (29). La population locale espère que la décentralisation constituera un cadre privilégié pour régler ces questions foncières, avec notamment l'application de la charte pastorale. Le dialogue à l'échelle communale devrait permettre aux autorités les plus proches de la population de se rencontrer, de négocier et de fixer ensemble les règles concernant les relations des communautés entre elles.

\section{CONCLUSION}

L'agriculture représente une véritable opportunité pour la fraction gouanine. Tout d'abord, elle constitue une garantie supplémentaire pour la sécurité alimentaire, en produisant des denrées directement consommables par l'homme, ou bien indirectement à travers la valorisation de fourrages par l'élevage. Par ailleurs, pour atténuer les conséquences des aléas climatiques et économiques, elle peut être considérée comme une diversification à l'élevage. Enfin, elle joue un rôle non négligeable dans le marquage des terres, fonction particulièrement importante dans le contexte actuel de pression foncière. Certaines familles gouanines ont ainsi élaboré des formes assez particulières d'intégration agriculture élevage avec le maintien du nomadisme conjugué aux activités agricoles (reposant notamment sur la constitution de stocks fourragers grâce au bourgou).

Néanmoins, le passage du nomadisme à la sédentarisation est un processus difficile : il constitue une rupture au niveau professionnel, mais plus encore au niveau du mode de vie. L'analyse de la situation met en avant la nécessité d'un cadre réglementaire approprié, dans un souci d'organisation et de concertation des hommes, et de gestion des ressources naturelles. Se dessine ici un enjeu fondamental de la décentralisation et de la mise en place de la charte pastorale dont les réussites dépendront de leurs modalités de mise en œuvre.

La majorité des interventions se concentre aujourd'hui au niveau de la frange fluviale. Or le phénomène de sédentarisation concerne seulement un quart de la fraction arabe étudiée. Ne faudrait-il pas donner la priorité aux populations majoritaires et soumises à davantage de risques qu'aux habitants de Téherdjé ? Il est nécessaire de replacer les dynamiques agricoles de la frange fluviale dans un ensemble géographique s'étendant aux espaces parcourus par les nomades, dans le Gourma, exploités ici par les Gouanines, mais aussi vers le nord dans le Haoussa, jusqu'à l'Azaouad.

\section{BIBLIOGRAPHIE}

1. ADAMOU A., 1999. La décentralisation au Niger : le cas de l'Aïr. In : Bourgeot A., éd., Horizons nomades en Afrique sahélienne. Sociétés, développement et démocratie. Paris, France, Karthala, p. 201-215.

2. ALARY V., MESSAD S., TACHE C., TILLARD E., 2002. Approche de la diversité des systèmes d'élevage laitiers à la Réunion. Revue Elev. Méd. vét. Pays trop., 55 : 285-297.

3. BARFIELD T.J., 1993. The nomadic alternative. Upper Saddle River, NJ, USA, Prentice Hall, 230 p.

4. BARRAUD V., MAHAMAT SALEH O., MAMIS D., 2001. L'élevage transhumant au Tchad oriental. Lyon, France, VSF, 137 p.

5. BAUDOUX L., 2003. La frange fluviale du Niger au Nord-Mali : points de convergence entre l'agriculture et l'élevage. Diagnostic agraire réalisé dans la zone de Téherdjé, $6^{\mathrm{e}}$ région, auprès de communautés sonraï, kel-tamacheq et arabe. Mémoire fin d'études, ISA Lille / Esat1-Cnearc, Montpellier, France, 110 p.

6. BENKAHLA A., FERRATON N., BAINVILLE S., 2003. Initiation à une démarche de dialogue. Etude de l'agriculture dans le village de Fégoun au nord de Bamako au Mali. Paris, France, Gret, 125 p.

7. BERNUS E., 1981. Touaregs nigériens. Unité culturelle et diversité régionale d'un peuple pasteur. Paris, France, Orstom, 507 p.

8. BONFIGLIOLI A.M., 1990. Pastoralisme, agro-pastoralisme et retour : itinéraires sahéliens. Cah. Orstom, Sér. Sci. Hum., 26 : 255-266.

9. BOUDET G., 1984. Manuel sur les pâturages tropicaux et les cultures fourragères. Paris, France, ministère des Relations extérieures, 267 p. (Coll. Manuels et précis d'élevage)

10. BOURBOUZE A., 2000. Pastoralisme au Maghreb: la révolution silencieuse. Fourrages, $161: 3-21$.

11. CLAUDE J., GROUZIS M., MILLEVILLE P., éds, 1991. Un espace sahélien : la mare d'Oursi, Burkina-Faso. Paris, France, Orstom, 210 p.

12. COCHET H., 2001. Crises et révolutions agricoles au Burundi. Paris, France, INA-PG / Karthala, 468 p. 
13. DIGARD J.P., LANDAIS E., LHOSTE P., 1993. La crise des sociétés pastorales. Un regard pluridisciplinaire. Revue Elev. Méd. vét. Pays trop., 46 : 683-692

14. DJAMA M., 1999. Producteurs pastoraux et commerce international L'évolution des rapports marchands en pays Nord-Somali. In Bourgeot A., éd., Horizons nomades en Afrique sahélienne. Sociétés, développement et démocratie. Paris, France, Karthala, p. 339-353.

15. EDLIN M., MILLEVILLE P., éds, 1989. Le risque en agriculture. Paris, France, Orstom, $619 \mathrm{p}$

16. FAO, 2002. Pastoralism in the new millennium. Rome, Italy, FAO, 96 p. (Anim. Prod. Health Paper, No 150)

17. HAMMEL L., 2001. Terroirs d'attache des pasteurs du Niger. London, UK, IIED, 26 p. (Dossier $n^{\circ} 102$ )

18. HESSE C., 2001. Gestion des parcours : qui en est responsable et qui y a droit? In : Tielkes E., Schlecht E., Hiernaux P., éds, Elevage et gestion de parcours au Sahel, implications pour le développement. CR atelier régional ouest-africain, Niamey, Niger, 2-6 oct. 2000. Beuren, Allemagne, Verlag Ulrich E. Grauer, p. 139-153.

19. HUMPHREY C., SNEATH D., 1999. The end of nomadism? Society, State and the Environment in Inner Asia. Durham, NC, USA, Duke University Press, $355 \mathrm{p}$.

20. KOBRICH C., REHMAN T., KHAN M., 2003. Typification of farming systems for constructing representative farm models: two illustrations of the application of multi-variate analyses in Chile and Pakistan. Agric. Sys., 76: 141-157

21. LANDAIS E., 1996. Typologies d'exploitations agricoles. Nouvelles questions, nouvelles méthodes. Econ. rurale, 236 : 3-15.

22. LANDAIS E., LHOSTE P., 1990. Les relations agriculture-élevage en Afrique intertropicale : un mythe techniciste confronté aux réalités du terrain. Cah. Orstom, Sér. Sci. Hum., 26 : 217-235.

\section{Summary}

Baudoux L., Kamil H., Moulin C.H. Development of Agropastoralism in a Nomadic Community Settled along the Niger River in Mali

In the region of Timbuktu in Mali, the droughts of the 197080 's and the Tuareg rebellion of the 1990's led nomadic populations to settlement and cultivation. At the beginning of a new project supporting agropastoral organizations, the NGO Vétérinaires Sans Frontières launched several studies to assess these changes. Thus, we studied the evolution of the agricultural system of an Arab community settled along the Niger River. Three farming systems were characterized. The transhumant agropastoralists (20\% of the families) are settled on the Niger banks. They grow rice and burgu grass in the flood plains. They rear small ruminant herds that do not however satisfy all family needs. The nomadic pastoralists (74\%) still specialize in livestock farming; livestock management is based on herd and family mobility in order to exploit Sahelian rangelands. At last, semi-nomadic agropastoralists $(6 \%)$ combine a settled way of life during the dry season on the river banks and nomadism during the rainy season, while salaried employees work in their fields. The study of the relationship between livestock farming and cropping, and of resources use can help shape development policies based on crop intensification on the river banks.

Keywords: Nomadism - Agropastoral system - Cropping system - Animal husbandry - Niger River - Mali.
23. LANDAIS E., LHOSTE P., MILLEVILLE P., 1987. Points de vue sur la zootechnie et les systèmes d'élevage tropicaux. Cah. Orstom, Sér. Sci. Hum., $23: 421-437$.

24. MAZOYER M., ROUDART L., 1997. Histoire des agricultures du Monde. Du néolithique à la crise contemporaine. Paris, France, Le seuil, 534 p.

25. MOULIN C.H., PLUVINAGE J., BOCQUIER F., 2004. Les relations entre agrandissement des troupeaux et changements de conduite: exemple des élevages d'ovins allaitants en Crau. Rencontres Rech. Ruminants, 11 : 145-148.

26. ORSINI J.P.G., LHOSTE P., BOUCHIER A., FAYE A., NIANG L., 1985. Une typologie d'exploitations agropastorales au Siné-Saloum, Sénégal. Revue Elev. Méd. vét. Pays trop., 38 : 200-210.

27. PLUVINAGE J., MOULIN C.H., 2004. Analyse de la diversité et de la dynamique des systèmes de production : propositions méthodologiques à partir d'études menées en régions d'élevage. In : Colloque Les systèmes de production : performances, évolutions, perspectives, Société française d'économie rurale, Lille, France, 18-19 nov. 2004. (http://www.sfer.asso. $\mathrm{fr} / \mathrm{sfer} /)$

28. SCOONES I., dir., 1999. Nouvelles orientations du développement pastoral en Afrique. Paris, France, Karthala / CTA, 362 p.

29. SWIFT J., 1999. Les écosystèmes dynamiques et I'administration du développement pastoral. In : Scoones I., éd., Nouvelles orientations du développement pastoral en Afrique. Paris, France, Karthala / CTA, p. 265-296.

30. THEBAUD B., 1995. Le foncier dans le Sahel pastoral. Situation et perspectives. In : Blanc-Pamard C., Cambrézy L., éds, Terre, terroir, territoire. Les tensions foncières. Paris, France, Orstom, Sér. Dynamique des systèmes agraires, p. 37-56.

31. THEBAUD B., 2002. Foncier pastoral et gestion de I'espace au Sahel. Peuls du Niger oriental et du Yagha burkinabé. Paris, France, Karthala, 318 p.

Reçu le 28.06.2004, accepté le 02.05.2005

\section{Resumen}

Baudoux L., Kamil H., Moulin C.H. Desarrollo agro pastoral en una fracción nómada establecida sobre los bordes del río Níger en Malí

En la región de Tombuctú en Malí, las sequías de los años 1970-80 y de la rebelión touareg de los años 1990 trajeron el sedentarismo de las poblaciones nómadas y el desarrollo de la agricultura. Con el fin de conocer mejor estas transformaciones, la ONG Veterinarios sin fronteras lanzó una serie de estudios coincidiendo con el inicio de un nuevo proyecto de apoyo a las organizaciones agro pastoriles. Se analizó así la evolución del sistema agrario de una fracción árabe, establecida sobre los bordes del río Níger. Se caracterizaron tres sistemas de producción. Los agro pastores trashumantes $(20 \%$ de las familias de la fracción) sedentarios sobre la margen fluvial. Cultivan el arroz y el burgú en las llanuras inundables y poseen pequeños hatos de rumiantes, insuficientes para satisfacer el conjunto de las necesidades de la familia. Los pastores nómadas (74\%) especializados, desde siempre, en la cría y han conservado un modo de conducta pastoril que reposa sobre la movilidad de los hatos y de las familias para realzar los trayectos saharianos. Finalmente, los agro pastores semi-nómadas $(6 \%)$ combinan la vida de sedentarios durante la estación seca, sobre el sitio de fijación al borde del río y el nomadismo durante la estación lluviosa, los trabajos de los campos siendo entonces asegurados por asalariados. El estudio de las relaciones agricultura-cría y el uso de los recursos permite discutir las políticas de desarrollo centradas sobre la intensificación agrícola sobre el borde fluvial.

Palabras clave: Nomadismo - Systema agropascicola - Sistema de cultivo - Ganadería - Río Niger - Malí. 